\title{
THE RESOLVED STELLAR HALO OF NGC 253*,
}

\author{
Jeremy Bailin ${ }^{1}$, Eric F. Bell ${ }^{1}$, Samantha N. Chappell ${ }^{1}$, David J. Radburn-Smith ${ }^{2}$, And Roelof S. De Jong ${ }^{3}$ \\ ${ }^{1}$ Department of Astronomy, University of Michigan, 830 Dennison Building, 500 Church Street, Ann Arbor, MI 48109, USA; jbailin@ umich.edu \\ 2 Department of Astronomy, University of Washington, Seattle, WA 98195, USA \\ ${ }^{3}$ Leibniz-Institut für Astrophysik Potsdam (AIP), An der Sternwarte 16, D-14482 Potsdam, Germany \\ Received 2011 February 8; accepted 2011 April 28; published 2011 June 30
}

\begin{abstract}
We have obtained Magellan/IMACS and Hubble Space Telescope (HST)/Advanced Camera for Surveys imaging data that resolve red giant branch stars in the stellar halo of the starburst galaxy NGC 253. The HST data cover a small area, and allow us to accurately interpret the ground-based data, which cover $30 \%$ of the halo to a distance of $30 \mathrm{kpc}$, allowing us to make detailed quantitative measurements of the global properties and structure of a stellar halo outside of the Local Group. The geometry of the halo is significantly flattened in the same sense as the disk, with a projected axis ratio of $b / a \approx 0.35 \pm 0.1$. The total stellar mass of the halo is estimated to be $M_{\text {halo }} \sim(2.5 \pm 1.5) \times 10^{9} M_{\odot}$, or $6 \%$ of the total stellar mass of the galaxy, and has a projected radial dependence that follows a power law of index $-2.8 \pm 0.6$, corresponding to a three-dimensional power-law index of $\sim-4$. The total luminosity and profile shape that we measure for NGC 253 are somewhat larger and steeper than the equivalent values for the Milky Way and M31, but are well within the scatter of model predictions for the properties of stellar halos built up in a cosmological context. Structure within the halo is seen at a variety of scales: there is small kpc-scale density variation and a large shelf-like feature near the middle of the field. The techniques that have been developed will be essential for quantitatively comparing our upcoming larger sample of observed stellar halos to models of halo formation.
\end{abstract}

Key words: galaxies: formation - galaxies: halos - galaxies: individual (NGC 253) - galaxies: spiral - galaxies: starburst - galaxies: structure

Online-only material: color figures, machine-readable table

\section{INTRODUCTION}

In the favored hierarchical model of galaxy formation (White \& Rees 1978), stellar halos are composed of the shredded remains of the smaller protogalactic clumps that assembled the larger galaxy (Searle \& Zinn 1978; Bullock et al. 2001). At radii approaching the virial radius, the dynamical time within the halo approaches the age of the universe. Stellar halos thus retain both spatial and kinematic information about this assembly history that is otherwise difficult to obtain.

The stellar halo of our own Galaxy has been extensively surveyed. Studies of high-velocity stars in the solar neighborhood and bright stars at large distances have revealed a halo that follows a power-law density distribution, a total luminosity of approximately $10^{9} L_{\odot}$, and abundant spatial, kinematic, abundance, and stellar population substructure (e.g., Helmi et al. 1999; Majewski et al. 2003; Yanny et al. 2003; Belokurov et al. 2007; Carollo et al. 2007; Bell et al. 2008, 2010; Xue et al. 2010; Cooper et al. 2010a). However, our position within the disk of the Milky Way makes it difficult to obtain a clear picture of the global structure of the halo. This is additionally complicated by the fact that different tracer populations must be used to determine the structure at different distances.

Some information has been obtained about the halos of external galaxies by searching for surface brightness features

\footnotetext{
* This paper includes data gathered with the $6.5 \mathrm{~m}$ Magellan Telescopes located at Las Campanas Observatory, Chile.

$\dagger$ Based on observations made with the NASA/ESA Hubble Space Telescope, obtained at the Space Telescope Science Institute, which is operated by the Association of Universities for Research in Astronomy, Inc., under NASA contract NAS 5-26555. These observations are associated with program 10523.
}

in deep imaging (e.g., Beck et al. 1982; Malin \& Hadley 1997; Zheng et al. 1999; Martínez-Delgado et al. 2008, 2010). These observations have identified prominent tidal streams that buttress the hierarchical model of galactic halo formation. However, the fundamental limits in such studies are flat-fielding errors and the brightness of the background sky, which make global halo properties and very faint surface brightness features inaccessible (de Jong 2008).

In order to overcome these difficulties, a new technique has recently been employed: resolving individual bright stars, particularly red giant branch (RGB) stars which are present in all stellar populations, in the halos of external galaxies. Within the past few years, knowledge of the halo of M31 has blossomed with this technique, including the discovery of a metallicity gradient, rich spatial and kinematic substructure, and a global profile that is remarkably similar to that of the Milky Way (Chapman et al. 2006; Kalirai et al. 2006; Ibata et al. 2007; McConnachie et al. 2009; Tanaka et al. 2010). Detections of halo stars in more distant galaxies are now beginning to be achieved, such as in individual small Hubble Space Telescope (HST)/Advanced Camera for Surveys (ACS) fields of NGC 5128 (Rejkuba et al. 2005) and M 81 (Durrell et al. 2010), and in NGC 891 using ground-based Subaru/Suprime-Cam data over a large field of view (Mouhcine et al. 2010).

One particularly interesting target is NGC 253. This nearly edge-on starburst galaxy, one of the largest galaxies in the "Sculptor group" (which is not truly a bound group, but rather a filament extended along the line of sight; Jerjen et al. 1998; Karachentsev et al. 2003), is of similar luminosity to the Milky Way and M31, allowing direct comparisons. Deep optical images have long shown an optical halo extending beyond its 
disk, with evidence for a shelf-like feature to the south of the disk (Beck et al. 1982; Malin \& Hadley 1997). Fitzgibbons (1990), in a photographic survey of the galaxy, also detected extended structure to the south of the galactic disk, particularly in the $I$ band. He found a total light profile in the halo that declines as a power law with an index of $\sim-2.5$ and $\sim-3.5$ in the $V$ and $I$ bands, respectively.

Resolved near-infrared stellar photometry of the southeast quadrant of NGC 253 was performed by Davidge (2010; see also Comerón et al. 2001, who detected blue main-sequence stars in the halo near the minor axis of the galaxy, most likely associated with the outflow from the central starburst). He detected an extended flattened distribution of asymptotic giant branch (AGB) stars extending out to $13 \mathrm{kpc}$ from the disk plane. Based on the similarity of the disk and halo $K$-band luminosity functions, which implies an extended star formation history, he concluded that these stars were deposited into the halo via the disruption of the galactic disk in an interaction. The data were not of sufficient depth to detect RGB stars, which would be far more numerous and trace stellar populations of all ages.

Theoretical models of galaxy formation in a $\Lambda \mathrm{CDM}$ universe have been used to predict the properties of stellar halos, usually with the assumption that the stars that are today found in the halo were accreted in the form of satellite galaxies that were subsequently tidally shredded (Bullock et al. 2001; Bullock \& Johnston 2005; Purcell et al. 2007; Cooper et al. 2010b). These models predict that halos contain several percent of the total stellar mass of the galaxy, and have very clumpy structure, particularly in the outer regions. They also predict significant galaxy-to-galaxy scatter in the total size and morphology of the halo structure (Bullock \& Johnston 2005; Bell et al. 2008; Johnston et al. 2008). While these properties qualitatively match observed galactic halos, quantitative measurements of a larger sample of halos is required in order to adequately test these models and distinguish between them.

Our goal is to test these models by obtaining resolved data of stellar halos in a larger, and therefore more representative, sample of galaxies. This will allow us to accurately determine stellar halo structural properties, and to investigate their correlation with the global parameters of the associated galaxies. The larger sample will help determine any scatter between systems due to a stochastic formation history. Because of the large degree of predicted and observed substructure, obtaining an unbiased view of the halo requires a large field of view that is only possible using ground-based data. However, identification and accurate photometry of stars in galaxies beyond the Local Group is far superior with $H S T$. We are therefore using a combination of HST and ground-based wide-field imaging.

In this paper, we present the first results of this program, a study of NGC 253, along with a detailed description of the analysis required to turn the data into quantitative global measures of halo structure. We adopt a distance modulus to NGC 253 of $m-M=27.71(D=3.48 \mathrm{Mpc})$ throughout, as determined using GHOSTS (Radburn-Smith et al. 2011), and consistent with but toward the upper end of distance estimates based on a variety of methods cataloged in the NASA Extragalactic Database ${ }^{4}$ (NED). Note that we use the term "halo" to refer to the stellar halo of the galaxy, not the dark matter halo, unless specifically stated otherwise.

\footnotetext{
4 http://nedwww.ipac.caltech.edu/
}

\section{DATA}

\subsection{Observations and Reduction}

\subsubsection{Magellan/IMACS Data}

The ground-based data were obtained at the Magellan 1 (Baade) $6.5 \mathrm{~m}$ telescope using the Inamori-Magellan Areal Camera and Spectrograph (IMACS), a mosaic of eight $2 \mathrm{k} \times 4 \mathrm{k}$ CCDs, in $f / 2$ configuration. This results in a 0.2 pixel $^{-1}$ plate scale. The field was centered $14^{\prime}$ south of the center of NGC 253 , and therefore included a significant fraction of the galactic disk within the $24^{\prime}$ unvignetted field of view. At the assumed distance of NGC 253, the field extends $30 \mathrm{kpc}$ from the galaxy center. On the nights of 2009 October 17 and 18, we obtained $1800 \mathrm{~s}$ in CTIO $I$ band and $2650 \mathrm{~s}$ in Bessel $V$ band. A further $1000 \mathrm{~s}$ were obtained in the $V$ band on the night of 2010 May 20. The seeing full width at half-maximum (FWHM) was approximately 1". $0-1$ ". 2 during the exposures. Landolt photometric standards were taken on these and other nights during the same runs.

Data were reduced using standard IRAF routines, ${ }^{5}$ assisted by scripts developed for reducing Maryland-Magellan Tunable Filter data. ${ }^{6}$ Note that although the IMACS documentation suggests using the column and row overscan regions instead of bias frames, we found that in the case of broadband imaging, saturated stars near the edges of the chips can bleed into the overscan regions, making them unusable. We therefore used bias frames that were obtained during the runs. Sky subtraction was complicated by the presence of the galactic disk in the field, which made it impossible to fit an accurate low-order surface to the sky. We therefore determined the sky level using a twodimensional median filter of width 101 pixels; this width was chosen to be large enough that photometry tests on individual stars found no bias, but small enough that the sky was flat to within $1 \%$ on large scales. This results in good image quality over most of the frame, but clearly invalidates the portion of the field that includes the disk of NGC 253, which contains smooth features on scales much larger than the filter width. However, as the goal of this work is to study the halo of the galaxy, this does not affect our results. An astrometric solution over the field was computed using the USNO A2.0 catalog (Monet et al. 1998). A third order polynomial solution was required in order that the astrometric residuals did not vary systematically across the field; the resulting solution achieved an rms scatter of 0.24 , comparable to the pixel size. Two sample regions of the final stacked and sky-subtracted $I$-band image are shown in Figure 1: one located $\sim 9.5(10 \mathrm{kpc})$ from the center of the galaxy and one located at $\sim 22^{\prime}$ ( $22 \mathrm{kpc}$ ), both approximately along the minor axis. The faint point sources that are abundant in the inner field but almost completely absent in the outer field are RGB stars in the halo of NGC 253. We ignore the very outermost part of the mosaic, which is compromised by the shadow of the guide star camera, whose location varied between exposures.

\subsubsection{GHOSTS}

We use data from the HST/GHOSTS survey (de Jong et al. 2007; Radburn-Smith et al. 2011) overlapping with our IMACS pointing for the purposes of carrying out our artificial star tests, and to test our inferred stellar densities (in the high stellar density regime). We use HST/ACS F606W and F814W data

\footnotetext{
5 IRAF is distributed by the National Optical Astronomy Observatory, which is operated by the Association of Universities for Research in Astronomy (AURA) under cooperative agreement with the National Science Foundation. 6 http://www.astro.umd.edu/ veilleux/mmtf/datared.html
} 

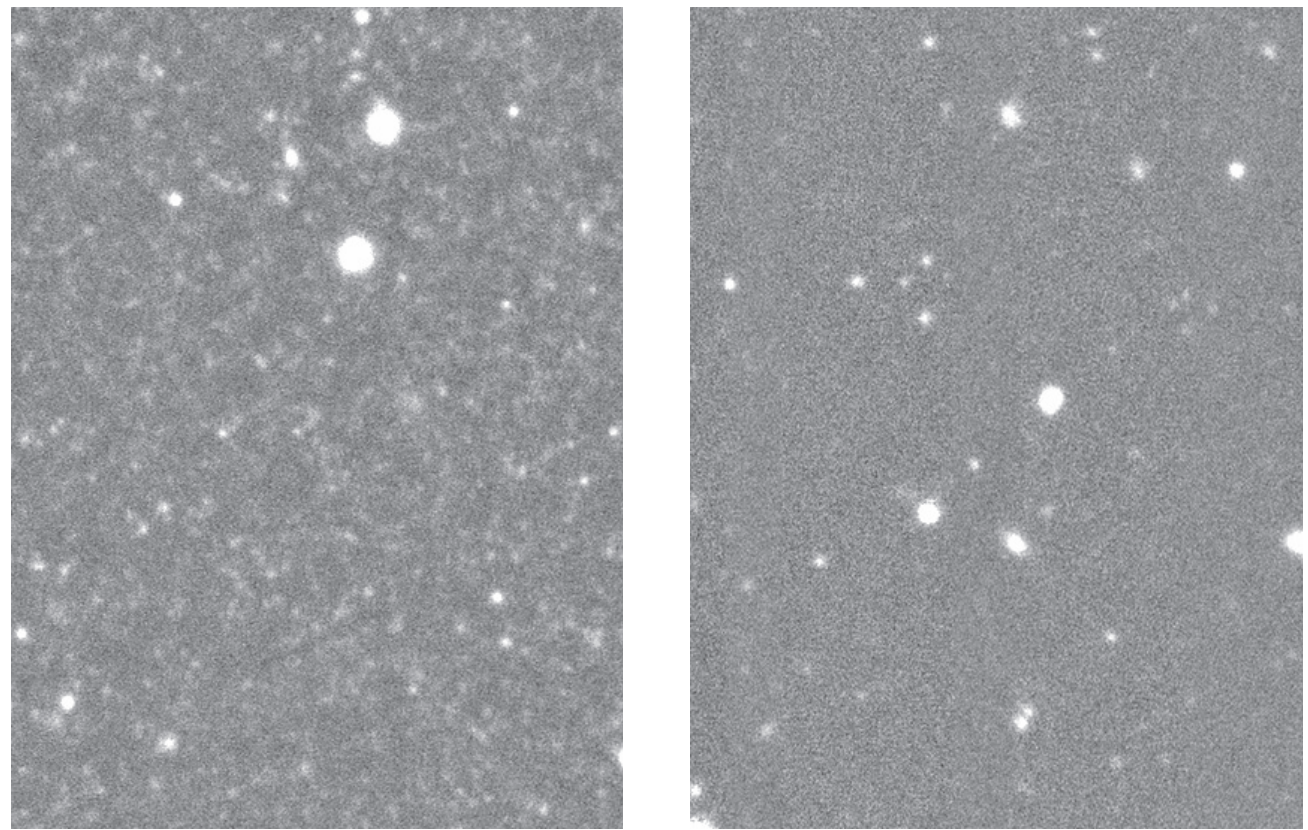

Figure 1. Left: sky-subtracted stacked IMACS $I$-band image of a $82^{\prime \prime} \times 110^{\prime \prime}$ region located $\sim 9.5(10 \mathrm{kpc})$ from the center of NGC 253 , along the minor axis. RGB stars within its stellar halo are clearly visible as faint point sources. Right: as in the left panel, for a region located $\sim 22^{\prime}(22 \mathrm{kpc})$ from the center of NGC 253 . Very few, if any, RGB candidates are visible.

for NGC 253's Fields 9 and 10 (Radburn-Smith et al. 2011), with exposure times of $2 \times 680 \mathrm{~s}$ in both F606W and F814W bands. Data were reduced and calibrated using the standard HST data reduction, and photometry was performed using the ACS module of DOLPHOT. ${ }^{7}$ Photometric parameters were converted to $V$ - and $I$-band magnitudes using the conversions presented in Sirianni et al. (2005). Typical uncertainties (random and systematic) are $<0.05 \mathrm{mag}$ at the $I<26$ limits of interest in this paper.

\subsection{Photometry}

Sources were found on the stacked IMACS I-band image using the IRAF DAOFIND task, with a threshold of three times the typical standard deviation of the background. Aperture photometry was performed using the IRAF APPHOT package on the stacked $V$ - and $I$-band images at the locations found by DAOFIND, using an aperture of radius 8 and 6 pixels, respectively, matched to the width of the point-spread function (PSF) to maximize the signal to noise. Aperture corrections were calculated in each band using five bright unsaturated relatively isolated stars. Magnitudes were de-reddened using the Galactic foreground extinction values cataloged in NED for NGC 253. The final catalog consists of all stars that have a positive measured flux in both bands.

Photometric calibration was performed using aperture photometry of standard stars in the Landolt fields SA98, SA101, and SA114 taken on the nights of 2009 October 17-19, using an aperture of 40 pixels, sufficient to enclose the entire flux of each star while not overlapping with neighboring stars. We fit a zero point, as well as airmass, color, and radial terms, ${ }^{8}$ to the residuals. The airmass term in the $I$ band and the color terms in both bands were found to be negligible, and so were assumed to be zero. The resulting photometry had an rms dispersion of

\footnotetext{
7 http://purcell.as.arizona.edu/dolphot/

8 The radial term accounts for the increase in solid angle subtended by pixels at larger radii.
}

$0.051 \mathrm{mag}$ in the $I$ band and $0.054 \mathrm{mag}$ in the $V$ band, constant over the 10 mag $<\mathrm{V}<15.3 \mathrm{mag}$ range of the standard stars. As will be seen later, such a level of photometric calibration accuracy is sufficient for our purposes, as it is smaller than other sources of systematic uncertainty.

A plot of the locations of all sources is shown in Figure 2. The points within the circle were used; this circular boundary is also used in the Monte Carlo simulations discussed in Section 3.2 to determine the effective surveyed area. Although the dithering pattern fully covered the chip gaps, the effective exposure time in the areas covered by chip gaps is lower and therefore the noise level is higher. This is reflected in the larger number of counts above the threshold in those regions, since the fixed threshold is no longer equal to $3 \sigma$. However, the excess counts are predominantly at the faint end, fainter than the brightness of the RGB candidates. The impact of the chip gaps is therefore small, although it is large enough that artificial structure along the horizontal chip gap is faintly visible in maps of RGB stars.

\section{COLOR-MAGNITUDE DIAGRAMS AND ARTIFICIAL STAR TESTS}

In this section, we present the color-magnitude diagrams (CMDs) of the ground-based and HST/ACS data, identify RGB stars in the data, and use artificial star tests to understand the relationship between the RGB star counts derived using the ground-based data and the intrinsic stellar population.

\subsection{Color-Magnitude Diagrams}

In Figure 3(a), we present the CMD of all recovered point sources within the main IMACS field. The bright blue vertical feature is primarily composed of pieces of the main body of NGC 253 that have been selected as stars by DAOFIND, along with the shredded diffraction pattern of foreground Milky Way main-sequence stars, and background disk galaxies. At the bottom, a wide feature that appears to be the RGB is seen. However comparison with the GHOSTS Field 10 data 


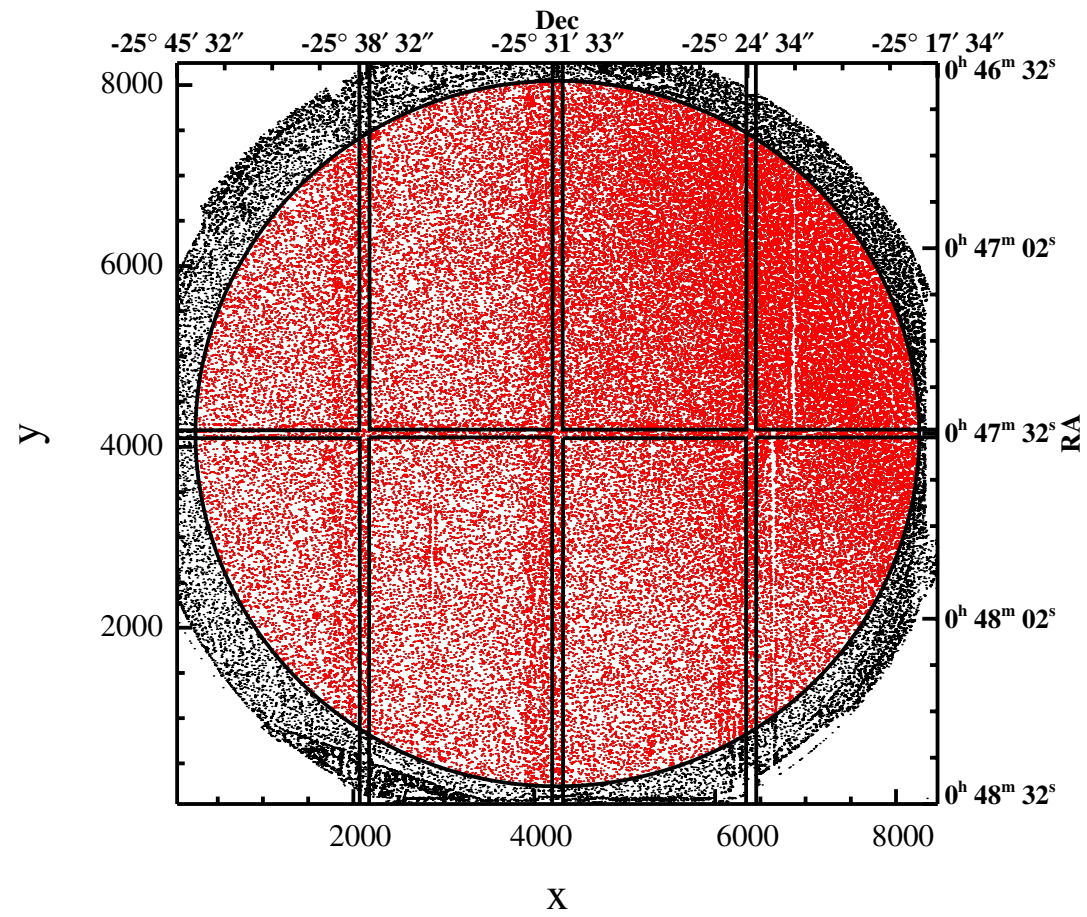

Figure 2. Locations of all stellar sources are plotted in pixel coordinates on the bottom left axes and angular coordinates on the sky on the upper right axes. Points within the circle are considered far enough from the edge of the field that they are usable (usable points are colored red in the online version of this figure). Outlines of the footprint of the IMACS chips for one particular dither position are shown by the rectangles.

(A color version of this figure is available in the online journal.)
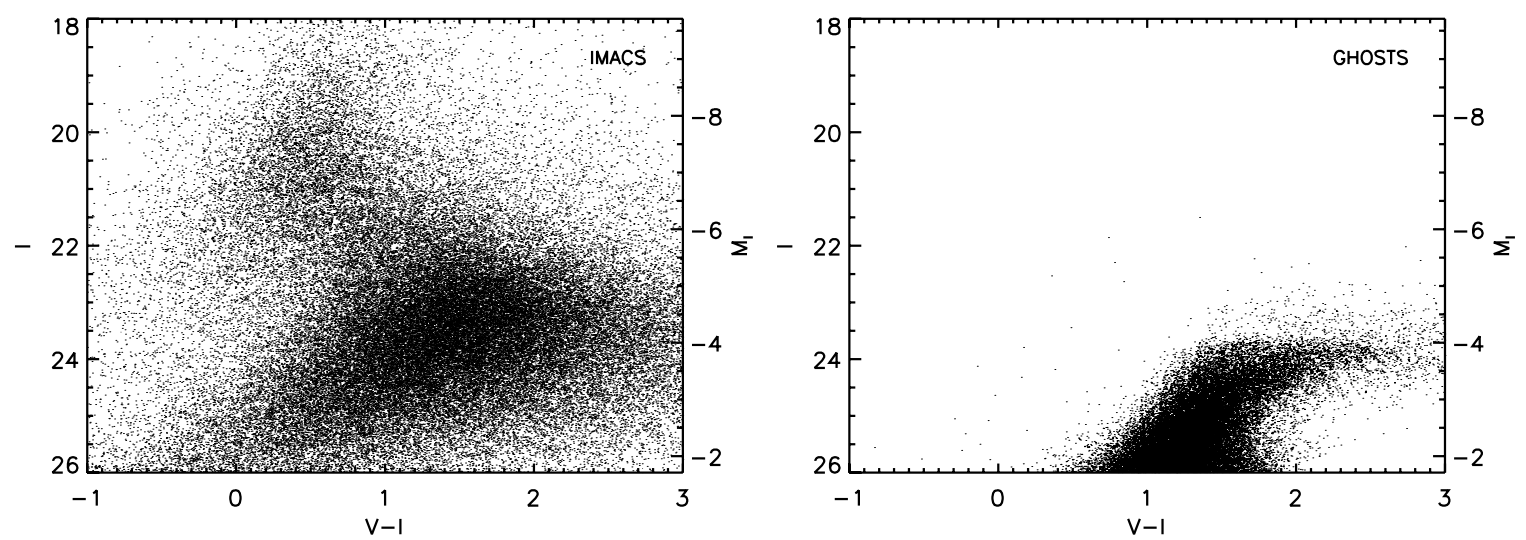

Figure 3. Color-magnitude diagrams of the Magellan/IMACS field (a) and GHOSTS HST/ACS field 10 (b) around NGC 253. The absolute magnitude scale on the right-hand axis assumes a distance modulus of $m-M=27.71$.

(Figure 3(b)) reveals that this putative RGB extends to much brighter magnitudes than in the HST/ACS data. Comparison of the IMACS and HST catalogs over their area of overlap reveals that the majority of "stars" detected in the IMACS catalog are blends of two or more stars. Therefore, while the detections in IMACS are indeed detections of RGB stars in the halo of NGC 253, interpretation of the number counts requires artificial star tests in order to determine the relationship between the numbers and properties of stars in the IMACS catalog and the true numbers and properties of the stellar population.

\subsection{Artificial Star Tests}

In order to test the photometric accuracy and recovery/ contamination rates of the tip of the red giant branch (TRGB) stars in NGC 253, we carried out a suite of artificial star tests. Artificial stars with $m_{I}<26$ were placed into a $1 \mathrm{k} \times 1 \mathrm{k}$ region in the outer parts of the $V$ - and $I$-band NGC 253 images with empirical PSFs generated from well-exposed stars. $V$ - and $I$-band apparent magnitudes were Monte Carlo sampled from the CMD of the HST/GHOSTS NGC 253 Field 10 pointing. The stars were distributed randomly across the region at eight different densities (labeled " $30 \mathrm{k}$ " through " $4000 \mathrm{k}$ " depending on the number of artificial stars that were added), spanning the observed range of RGB star densities seen in the IMACS image and in the GHOSTS data. The images were then output and processed by the photometric pipeline in the same way as the data.

Photometry was performed on the artificial star frames exactly as for the original frames. CMDs generated from the input magnitudes and from the output photometry on the artificial star frames for three sample artificial star tests (the 30k, 700k, and $4000 \mathrm{k}$ tests) are shown in Figure 4. As the stellar density increases, the RGB stars become blended and are detected at 


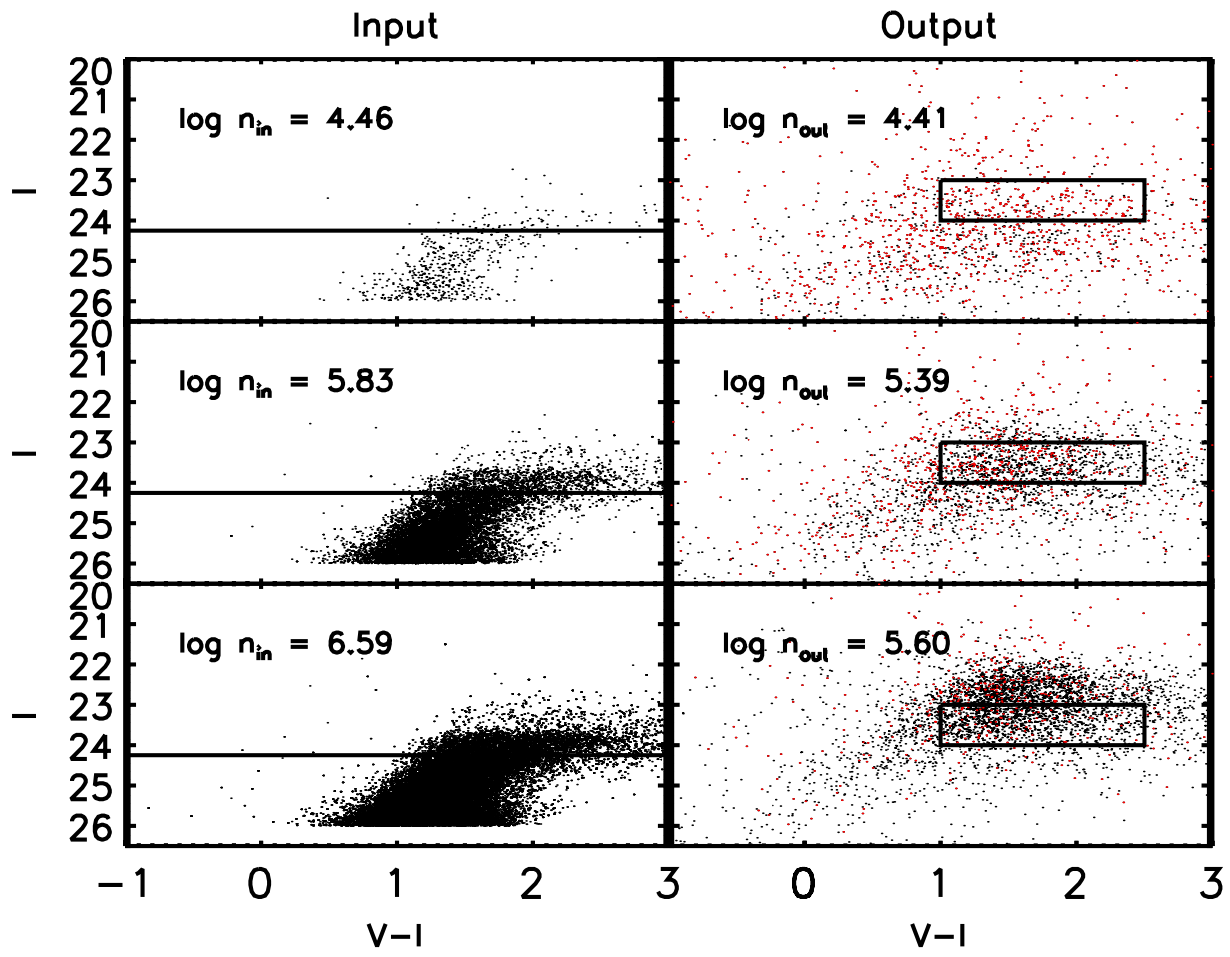

Figure 4. Artificial star CMDs. The left column shows input magnitudes of the artificial stars that were placed in the artificial star frames, while the right column shows the CMDs determined by performing photometry on the output artificial star frames. In the output CMDs, red points are those located within 0 .' 5 of a star in the original image. The rows correspond to injecting 30k (top), 700k (middle), and 4000k (bottom) artificial stars. The input and output density of stars is given in each panel, in number per square degree. The lines in the left column and the boxes in the right column denote the TRGB magnitude threshold and the boundaries of the RGB selection box, respectively.

magnitudes significantly brighter than those of individual stars. This results in a CMD exactly like the one observed if the majority of our sources are found in regions of high density.

In order to determine the number density of stars at the TRGB, we have compared the number of input RGB stars above a threshold of $I<24.25$ (corresponding to an absolute magnitude of $M_{I}<-3.46$ at the distance of NGC 253) to the number density of output detections within a box extending from $I=23$ to 24 , and from $V-I=1.0$ to 2.5 . This box lies entirely above the input threshold in order to better capture the bulk of the halo stars at the typical densities of our data (see Figure 4). We have tested several different criteria for both the TRGB threshold and the magnitude boundaries of the selection box, ranging by $\pm 0.5 \mathrm{mag}$. We find that this introduces $\sim 20 \%$ changes in the derived number densities once they are normalized by the expected number of stars above each TRGB threshold for an old metal-poor population, as discussed in Section 5.

The relationship between the input number density above the TRGB threshold and the output number density within the selection box is shown as the right-hand (high-density) dashed line in the upper panel of Figure 5, while their ratio (i.e., the recovered fraction) is shown in the bottom panel. A problem is immediately apparent: because there are 183 detections within the RGB selection box in the "blank field" to which the artificial stars were added (a combination of background galaxies and real RGB stars in the stellar halo), more stars are detected than were added at low densities. This is a property of the baseline image, not of our ability to detect stars, and so is artificial. One could simply subtract 183 from the number of output stars (shown as the left-handed dashed line in Figure 5), but this is also incorrect: at high densities, we approach the confusion-limited regime, and the original detections have mostly been covered up by artificial

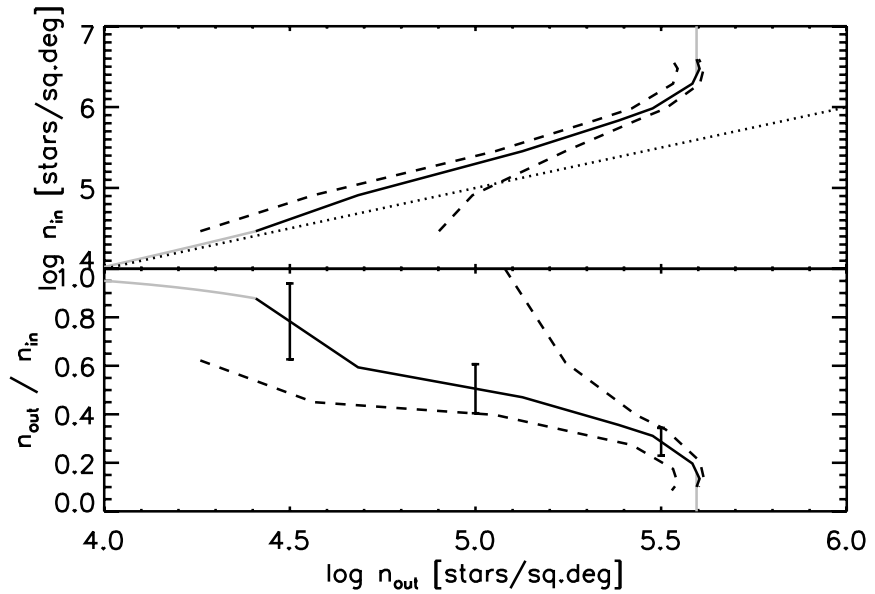

Figure 5. Results of the artificial star tests. The $x$-axis gives the density of stars recovered within the RGB selection box in the artificial star frame, while the $y$-axis gives the density of artificial stars injected into the artificial star frames that lie above the TRGB threshold (top) and the ratio of output to input stars (bottom). The dotted line in the upper panel denotes $n_{\text {in }}=n_{\text {out }}$. The right dashed line shows the case where no correction is made for the presence of stars in the baseline image. Subtracting the density of stars found in the original image gives the left dashed line, while subtracting only the stars in the original image that are located within 0.5 of a star in the artificial star frame gives the adopted solid line. The gray lines show the adopted extrapolations. The error bars in the bottom panel indicate the $20 \%$ uncertainty due to the choice of a particular TRGB magnitude threshold and CMD selection box.

stars and are therefore no longer detected. A better approach is to compare the locations of the detections in the artificial star frames to the locations of the original detections in the blank field. We performed a globally optimized one-to-one match of the closest original detection to each detection in the artificial 
star frame, and omitted any whose best match was within 0.'5. This results in the black solid line in Figure 5. The fraction of blank field sources that are omitted, $f_{\mathrm{bg}, \text { omit }}$, is a useful measure of the degree to which individual sources are likely to be covered up.

At the confusion limit, the number of output detections saturates, and even begins to decrease as the majority of the blends become brighter than the selection box. We therefore set a threshold of $\log n_{\text {out }}=5.60$, at which we can only set a lower limit on the true density $\left(\log n_{\text {in }} \geqslant 6.39\right)$, shown as the right-hand vertical gray line in Figure 5. Fortunately, very little of the data reach this limit. At the low density end, we note that the recovered fraction approaches unity, and so we make the ansatz that the recovered fraction has the form of a decaying exponential at densities below that of our 30k artificial star test; this results in the gray line extending to the left, which we use to extrapolate to lower densities. Again, most of our data lie above the point where we begin using this extrapolation, at $\log n_{\text {out }} \leqslant 4.39\left(\log n_{\text {in }} \leqslant 4.46\right)$. Our final correction from the artificial star test is therefore the combination of the solid black and gray lines in the top panel of Figure 5. Interpolation is performed linearly between $\log n_{\text {out }}$ and $\log n_{\text {in }}$. The error bars in the bottom panel indicate the $20 \%$ uncertainty due to the choice of TRGB threshold magnitude and CMD selection box.

Note that in order to convert number counts to densities, we required the effective area of the observed region. This is also required in order to calculate the densities in annuli, sectors, pixels in a density map, and the footprints of the GHOSTS pointings. Whenever the effective area was required, Monte Carlo simulations were performed. $N_{\mathrm{MC} \text { tot }}=10^{7}$ points were randomly sampled uniformly over a patch of the unit sphere of solid angle $\Omega_{\mathrm{MC} \text { tot }}=0.2088 \square^{\circ}$ encompassing the entire IMACS field. Within any given region, we counted the number of Monte Carlo points lying within that also lie within the field where stars were chosen (i.e., within the circle in Figure 2), $N_{\mathrm{MC}}$, and also the number of bad pixels, $N_{\text {bad }}$. The bad pixels included not only pixels formally counted as bad during the data reduction, but also regions that were completely devoid of detections due to bleed trails or the presence of a bright foreground star. We assume that the effective solid angle of each bad pixel is $\Omega_{\text {bad }}=0.04 \square^{\prime \prime}$, as given by the formal IMACS $f / 2$ plate scale; the true effective area varies over the field, but this effect is small relative to the two order of magnitude variation in the number density of RGB stars across the field, particularly given that the fraction of bad pixels is small. The total solid angle of the region is then given by

$$
\Omega_{\text {region }}=N_{\mathrm{MC}} \frac{\Omega_{\mathrm{MC} \text { tot }}}{N_{\mathrm{MC} \text { tot }}}-N_{\text {bad }} \Omega_{\mathrm{bad}} .
$$

\subsection{Background Galaxy Subtraction}

Some fraction of the detections must be due to background galaxies. To assess the impact of this background, we have used a control field that was created using $V$ and $I$-band imaging data taken using the Wide Field Imager on the ESO/MPG La Silla $2.2 \mathrm{~m}$ telescope of the Extended Chandra Deep Field South (see Gawiser et al. 2006). These calibrated images cover a slightly larger field of view than the IMACS data, and are somewhat deeper and have a smaller PSF $\left(0^{\prime} .9\right)$. The data were resampled onto 0.2 pixels (to match IMACS), convolved with the correct Gaussian to bring the FWHM to 1".04 and 1".2 for $I$ band and $V$ band, respectively, rescaled to the same zero point as the IMACS data, and sky noise was added to bring the rms of the image into agreement with the IMACS data for NGC 253. We then ran the images through our pipeline for star detection and photometry, and used the output photometric catalog as our "control field." The density of sources within the RGB selection box in the control field is $\log n_{\text {control }}=4.27 \square^{\circ-1}$. We scale this density by $f_{\mathrm{bg} \text {,omit }}$ before subtracting it; at densities below that of the 30k artificial star frame we subtract the full control density. The background counts are much smaller than the densities we derive over much of the halo, but are comparable to the densities in the outermost regions of the field.

\section{HALO STRUCTURE}

\subsection{Profiles \\ 4.1.1. Radial Profile}

The IMACS field was divided into radial bins of width $4 \mathrm{kpc}$, excluding the region within $5 \mathrm{kpc}$ of the disk plane in order to avoid thin and thick disk stars. These annuli were further subdivided into angular sectors of azimuthal extents $\Delta \theta=20^{\circ}$, in order to determine the structure of the halo at a given radius (different azimuthal bin sizes ranging from $16^{\circ}$ to $40^{\circ}$ were also tested, with no significant differences in our conclusions). The top right inset in Figure 6 shows the sectors; azimuth is defined with $\theta=0^{\circ}$ along the major axis to the northeast (upper left in the figure) and increasing counterclockwise.

The radial projected density profile is shown in the main panel of Figure 6. The error bars are the sum, in quadrature, of the Poisson error in the number of detected stars in each bin and the $20 \%$ uncertainty due to our choice of TRGB threshold and CMD selection box (see Section 3.2). The profile is well fit by a broken power law, of inner slope $n=-1.0 \pm 0.4$ and outer slope $n=-4.0 \pm 0.8$ (where the quoted error is the formal uncertainty in the fit, which is an underestimate; see below), with a break at $14.9 \mathrm{kpc}$. As demonstrated later, the break between these power laws is not due to an intrinsic change in the distribution of halo stars, but is rather an artifact of using circular annuli to describe a distribution that is intrinsically flattened. The IMACS counts within the GHOSTS fields are shown as the triangles, while the $H S T /$ ACS counts are shown as the squares. The difference between the triangles and squares gives an indication of the systematic uncertainty, $\sim 0.2$ dex, which dominates over the statistical errors shown in the profiles. This is discussed in more detail in Section 4.3. Given the size of this systematic uncertainty, a more realistic error for the slope of the radial power law can be obtained by shifting the inner points up by $0.2 \mathrm{dex}$; doing this changes the slope of the inner power law to $n=-1.6$ and the slope of the outer power law to $n=-5.3$. Although it appears that the IMACS counts within the outer GHOSTS field (Field 10) are much higher than the counts at adjacent radii, this is because the radial profile averages over all azimuths, while the GHOSTS field is at one specific azimuth. Indeed, as shown by the azimuthal profiles in the bottom left inset of Figure 6 (also shown as the gray lines with error bars on the main plot; these gray points are offset horizontally with azimuth for clarity), there is a strong trend in density with azimuth for each radial bin. The IMACS counts in the GHOSTS field are fully consistent with the counts in the same radial bin when this trend is taken into account. Overall, the almost uniform positive gradient in the azimuthal profiles indicate that the counts toward the galactic plane (at larger azimuth, which is defined to increase counterclockwise) are significantly higher than along the galactic minor axis at the same radius. This is strong evidence that the halo profile is not 


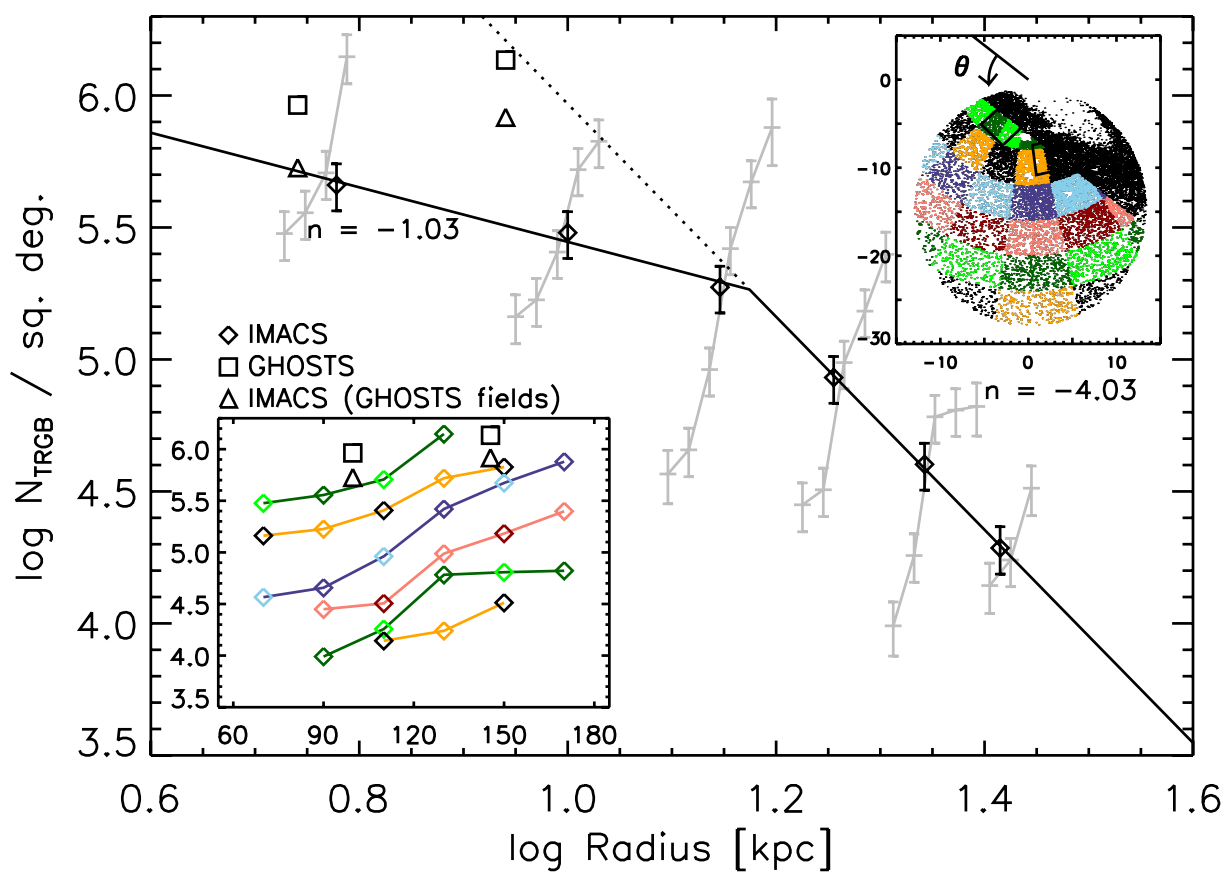

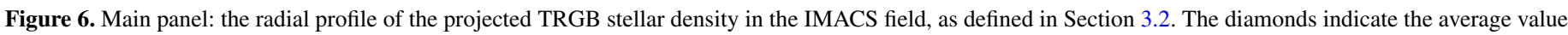

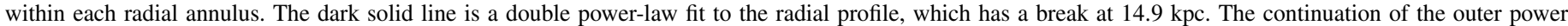

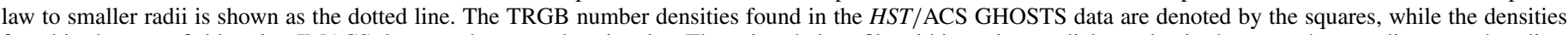

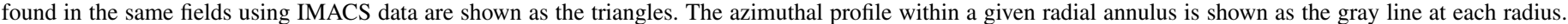

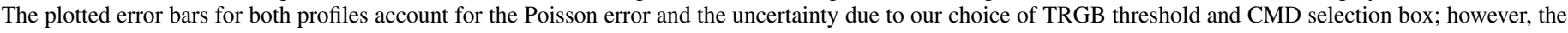

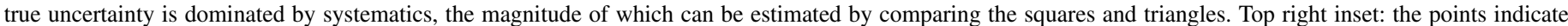

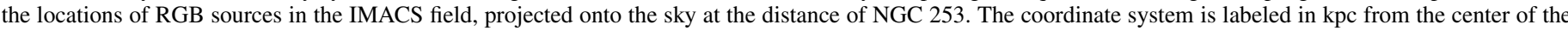

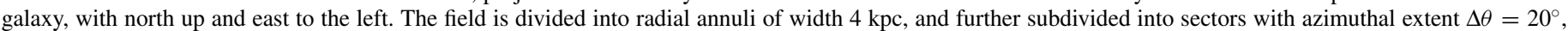

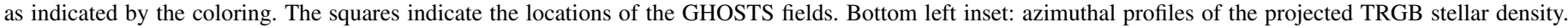

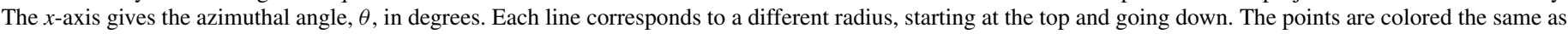

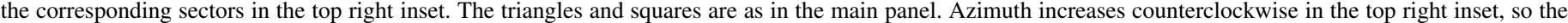
rightmost angular sector in this panel is the one closest to the disk plane.

spherically symmetric, but is rather flattened in the direction of the disk. Another possibility is that we are not seeing a true stellar halo, but rather a very extended thick disk, which would necessarily have a very flattened distribution (see discussion in Section 4.1.3).

\subsubsection{Elliptical Radial Profile}

The azimuthal trend of the halo density in Section 4.1.1 strongly indicates that the stellar halo of NGC 253 is flattened. Therefore, in order to determine an accurate radial profile, we must adopt "annuli" that mimic the intrinsic distribution of the stars. In this section, we adopt elliptical annuli aligned with the stellar disk. For the elliptical axis ratio, we have tested values ranging from the observed disk axis ratio of $b / a=0.25$ (Pence 1980; Koribalski et al. 2004), up to $b / a=0.5$. In the azimuthal direction, we adopt an angular coordinate $\phi$ defined by

$$
\tan \phi=\frac{r_{\text {maj }}}{r_{\min }} \frac{b}{a},
$$

where $r_{\text {maj }}$ and $r_{\text {min }}$ are the projections of the radial vector along the major and minor axes, respectively. If the halo distribution were perfectly disk-like, angular sectors spaced uniformly in $\phi$ would correspond to equal volumes. Our results are qualitatively unchanged if we instead adopt sectors that are spaced uniformly in the true projected azimuth $\theta$. The elliptical annuli had minor axes spaced by $4 \mathrm{kpc}$, which were further separated into sectors with azimuthal extent $\Delta \phi=8^{\circ}$, as shown in Figure 7 ( $\Delta \phi$ values from $4^{\circ}$ to $10^{\circ}$ showed similar behavior).
The radial halo profile in elliptical annuli with axis ratios $b / a=0.35$ is shown in the main panel of Figure 7. A single power law with a slope of $-2.8 \pm 0.3$ provides an acceptable fit to the data. This further demonstrates that the break in the power law seen in Figure 6 is entirely an artifact of the assumed geometry: the circular annuli coupled with the $5 \mathrm{kpc}$ inner cutoff probe different ranges of elliptical annuli as a function of radius. Shifting the inner points up by $0.2 \mathrm{dex}$, as in Section 4.1.1, steepens the slope to $n=-3.4$.

The azimuthal profiles are shown in the bottom left inset of Figure 7. Unlike with the circular annuli, the densities do not show a systematic gradient, again demonstrating the flattening of the halo. However, the azimuthal profiles are far from flat, and exhibit a strong peak at intermediate azimuths, $\phi \sim 100^{\circ}$. This could either indicate that the halo isophotes are smooth but "boxy," or could be an indication of discrete structure within the halo. The fact that the peak in azimuth is broad, rather than being confined to a single bin, indicates that it is not an artifact of the chip gap (which runs through many of the bins where the density peaks, particularly at large radius) or a manifestation of a thin tidal stream; it must be due to an extended or smooth feature. The shelf seen in the deep optical photometry of Malin \& Hadley (1997) extends in this direction, and it is likely that our azimuthal peak corresponds to this structure.

The choice of axis ratio $b / a=0.35$ was chosen by eye to minimize the variation of the azimuthal profiles. Values ranging from the disk ellipticity of $b / a=0.25$ to the value of $b / a=0.4$ that Davidge (2010) estimates for the stellar halo all provide reasonable, though somewhat poorer, descriptions. 


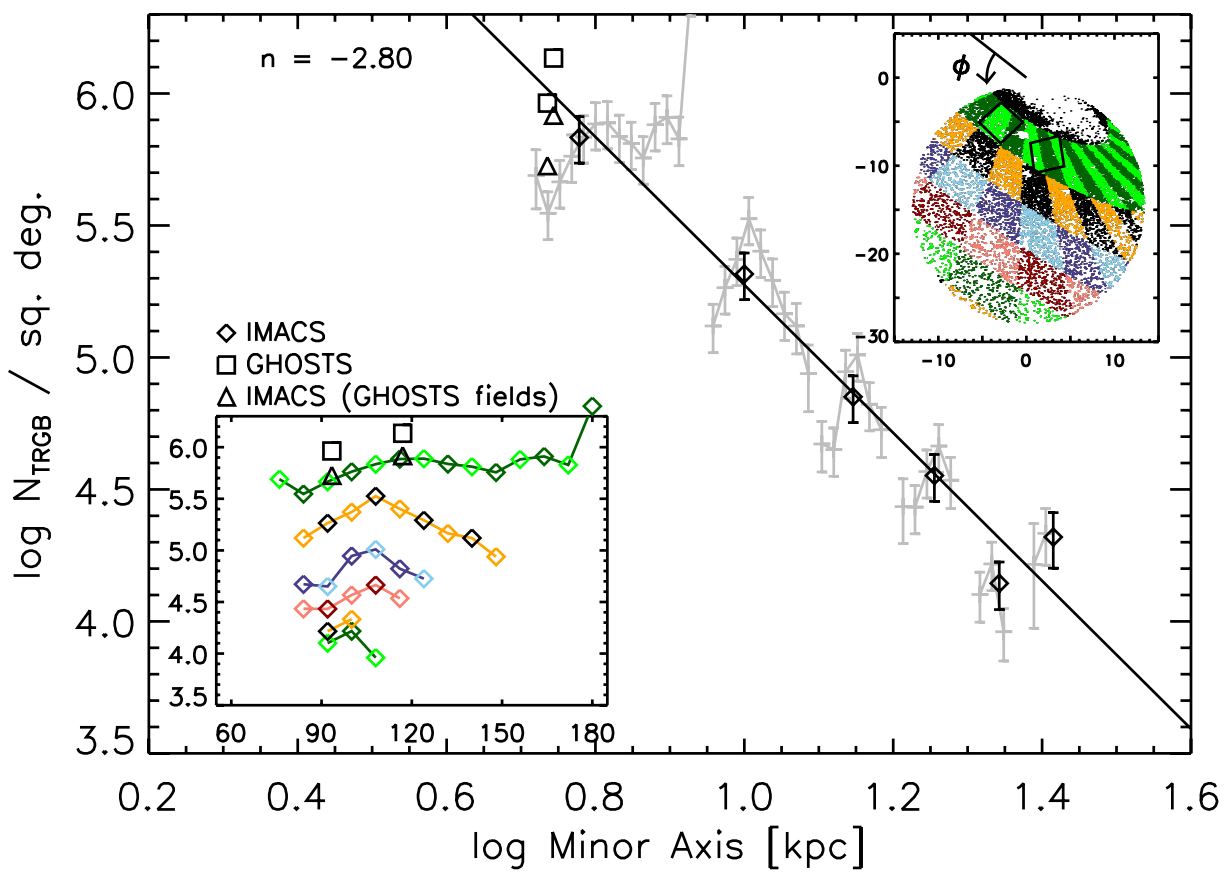

Figure 7. As in Figure 6, but using elliptical annuli of axis ratio $b / a=0.35$, with minor axis widths of $4 \mathrm{kpc}$ and elliptical sectors with azimuthal extents of $\Delta \phi=8^{\circ}$. A single power law is fit to the radial profile. The $x$-axis of the bottom left inset gives the angular coordinate, $\phi$, in degrees.

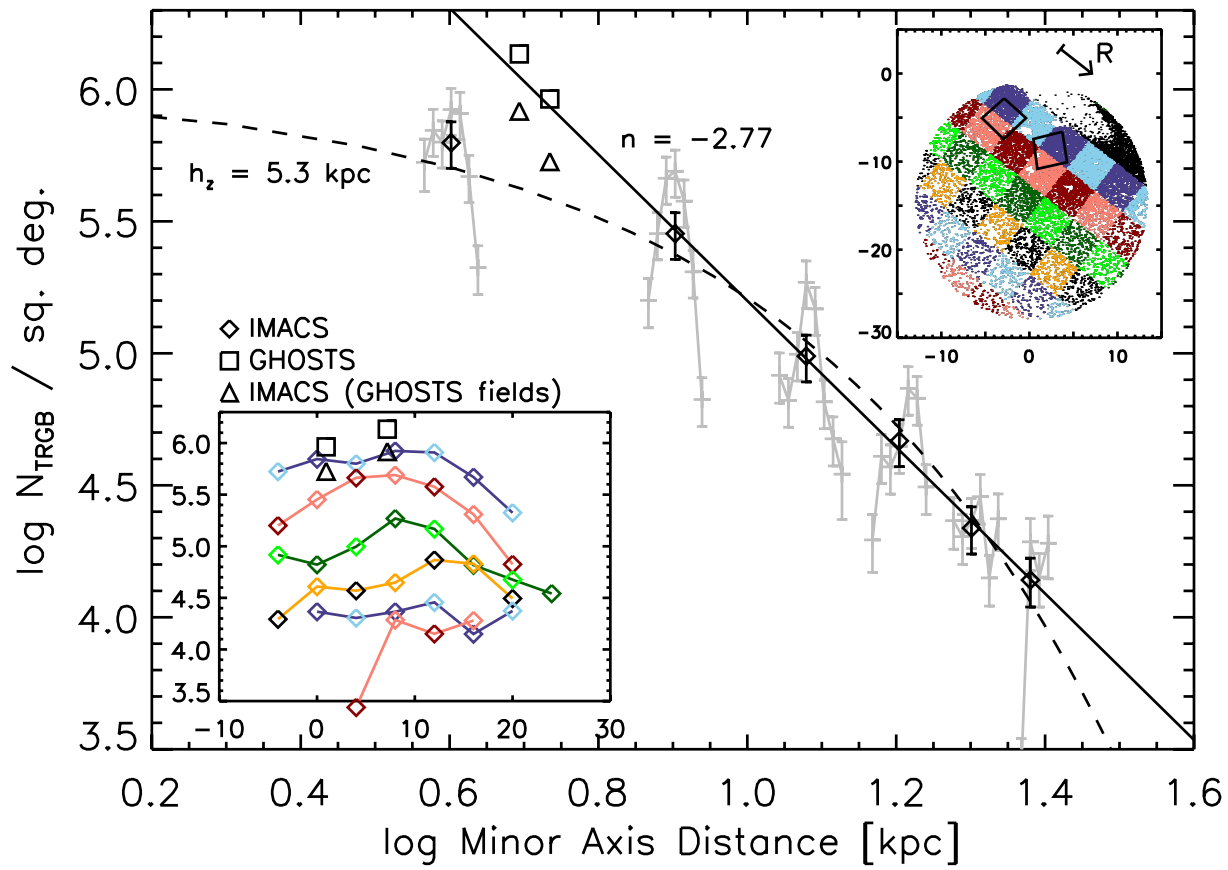

Figure 8. As in Figures 6 and 7, for bins of width $4 \mathrm{kpc}$ perpendicular to the disk and $4 \mathrm{kpc}$ along the disk major axis. The $x$-axis in the lower left inset indicates the distance in kpc along the major axis from the center of the galaxy. The solid line is a power-law fit to all but the innermost point, which is somewhat compromised by incompleteness within the disk. The dashed line is an exponential fit to the same data points, with scale height $h_{z}=5.3 \mathrm{kpc}$.

\subsubsection{Vertical Profile}

In the previous section, we found that the geometry of the halo must be quite flattened, being well fit on ellipses that are almost straight lines parallel to the disk over much of the field. It is therefore natural to ask whether a pure disk description, with the density depending only on distance from the galactic plane, provides a better description. We have constructed a vertical profile, calculated the density within vertical slices of $\Delta z=4 \mathrm{kpc}$ and further subdivided into rectangles of length
$4 \mathrm{kpc}$ in the direction parallel to the major axis, as shown in the top right inset of Figure 8 (major axis subdivisions ranging from 2 to $8 \mathrm{kpc}$ gave similar results). The innermost vertical sheet is somewhat compromised by incompleteness within the disk near the minor axis, and so the density of this bin was not used when fitting the density profile. The shapes of the ellipses and of the vertically stratified sheets are not too dissimilar over much of the field, and so it is not surprising that both the quality and slope $(-2.8 \pm 0.2$, steepening to -3.3 if the inner points are systematically low) of the best-fit power law are similar to the 
elliptical case. The density variation parallel to the major axis within each layer is also similar to the angular variation within the elliptical annuli.

Given that a description where the density varies primarily in the direction perpendicular to the disk is acceptable, it is natural to wonder whether the extended stellar structure we have detected is truly the stellar halo, or is rather a thick disk. This is pertinent given that M31, the galaxy whose outer regions are the best studied, shows evidence for both an extended rotating disklike component (Ibata et al. 2005) and a thick disk of estimated scale height $2.8 \mathrm{kpc}$ (Collins et al. 2011). In order to test this hypothesis, we have fitted a vertical exponential to the density structure we have detected (again omitting the innermost point), shown as the dashed line in Figure 8. An exponential is found to fit the data as well as the power law, but with an extremely largescale height of $h_{z}=5.3 \pm 0.5 \mathrm{kpc}(4.5 \mathrm{kpc}$ if the inner points are moved systematically higher). While we cannot rule out this possibility with the present data, we believe that a power-law halo is a more likely explanation for the following reasons.

1. Such a large-scale height is significantly larger than that of other stellar disks of comparable galaxies. While it is only 1.9 times larger than the $2.8 \mathrm{kpc}$ thick disk scale height estimated by Collins et al. (2011) for M31, these authors did not directly measure the scale height but rather assumed that the scale height varies with scale length in the same manner as the sample of low surface brightness galaxies studied by Yoachim \& Dalcanton (2006). The large thick disk scale height inferred for M31 is thus a direct consequence of its long disk scale length. NGC 253, on the other hand, has a much smaller scale length; estimates based on surface photometry at wavelengths from the optical through far infrared place the thin disk scale length between 1.5 and $2.6 \mathrm{kpc}$, once scaled to our adopted distance of $D=3.48$ Mpc (Pence 1980; Forbes \& Depoy 1992; Radovich et al. 2001). Adopting a thin-to-thick disk scale length ratio of 1.3 and the thick disk scale height-to-scale length ratio of 0.35 (Collins et al. 2011, based on Yoachim \& Dalcanton 2006), the expected thick disk scale height is between 0.7 and $1.2 \mathrm{kpc}$, many times smaller than what we derive. The derived scale height would require a very large vertical velocity dispersion to maintain; for a stellar mass surface density in the disk of $75 M_{\odot} \mathrm{pc}^{-2}$ (as for the Milky Way at the solar circle, Binney \& Tremaine 2008; this is a reasonable assumption given the similar total luminosity and disk scale length of the two galaxies), $\sigma_{z}=104 \mathrm{~km} \mathrm{~s}^{-1}$, making it essentially a pressure-supported structure.

2. Both IMACS (GHOSTS fields) data points lie above the extrapolation of the exponential fit.

3. The apparent quality of the exponential fit comes mainly from the innermost point, but this point is likely an underestimate due to incompleteness.

This issue can be resolved with more data that are deeper and cover a larger area; new HST GHOSTS data on NGC 253 and other galaxies are being analyzed to address the outer shape and profile of extended halo components.

\subsection{Density Map}

A map of the derived density of TRGB stars in the halo of NGC 253 is presented in Figure 9. This map was produced by binning the stars into pixels $1 \mathrm{kpc}$ on a side, and performing the background subtraction and artificial star correction. The image was then stretched using histogram equalization to

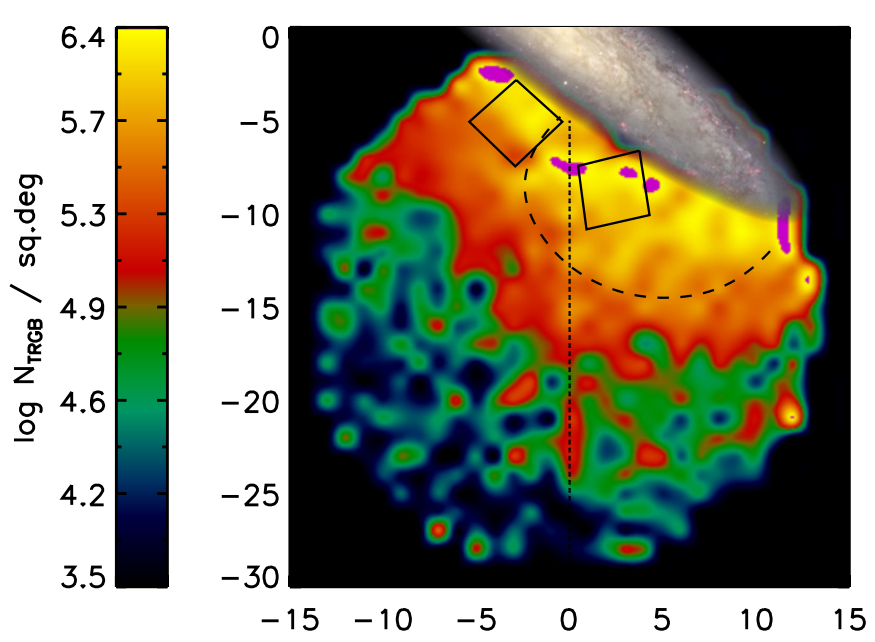

Figure 9. Map of the density of TRGB stars in the halo of NGC 253. The map is oriented with north up and east to the left. The $x$ - and $y$-axes are labeled in $\mathrm{kpc}$ from the center of the galaxy. Magenta indicates where the derived density is a lower limit on the true density. The GHOSTS pointings are denoted with the black squares, the location of the shelf is shown by the dashed curve, and the vertical dotted line denotes the horizontal chip gap, where the counts are artificially inflated. The dark ellipse at the top is the main body of the galaxy, where individual sources can no longer be distinguished; an optical image of the galaxy, to scale, has been overlaid for reference (image courtesy of T. A. Rector/University of Alaska Anchorage, T. Abbott and NOAO/AURA/ $\mathrm{NSF}$ ). The map was generated using $1 \mathrm{kpc} \times 1 \mathrm{kpc}$ pixels and then smoothed using the minimum curvature surface. Note that the mapping between the color scale and density value has been chosen to enhance visibility, so attention should be paid to the labels on the color bar, which give the logarithm of the TRGB number density per square degree.

enhance visibility, smoothed using the minimum curvature surface algorithm (Franke 1982), and resampled to a resolution of $0.1 \mathrm{kpc}$. The main body of the galaxy, where we cannot reliably detect stars, is apparent as the dark ellipse at the top (an optical image of the galaxy is overlaid, for reference), while the GHOSTS pointings are denoted by the black squares. Magenta indicates regions where the derived density reaches the saturation level in the IMACS data and we are therefore only able to place a lower limit on the true stellar density at these points.

A few features are notable in this map. First and foremost, the stellar density falls off strongly with radius from the galaxy, demonstrating that the stars belong to NGC 253. Secondly, the isopleths of constant stellar density are ellipses whose axis ratio is similar to or somewhat larger than that of the observed galaxy, as anticipated from Section 4.1.2. Thirdly, there are some large-scale substructures, chiefly a shelf-like feature near the center of the field (shown schematically by the dashed curve; see also Malin \& Hadley 1997, Figure 4). Finally, there is a small-scale pixel-to-pixel variation in the density. None of these features appears to be associated with any overdensity of resolved background galaxies. Note that the vertical feature at $x=0$ extending from $y=-20$ to $-25 \mathrm{kpc}$ lies right along the chip gap (shown with the dotted line), and is therefore most likely an artifact.

In Figure 10, we show the residuals between the density map and the best-fit power laws, expressed as the logarithm of the ratio. The left-hand panel shows the result using the radial power law. The systematic trend for the residuals to be negative on one side and positive on the other side demonstrates again that a simple radial power law is a poor description of the halo, i.e., it is not spherically symmetric. In the middle panel, the elliptical power-law fit is used. In this case, the residuals show 

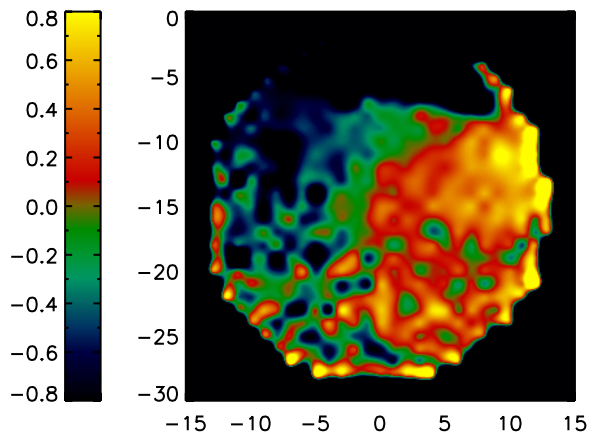
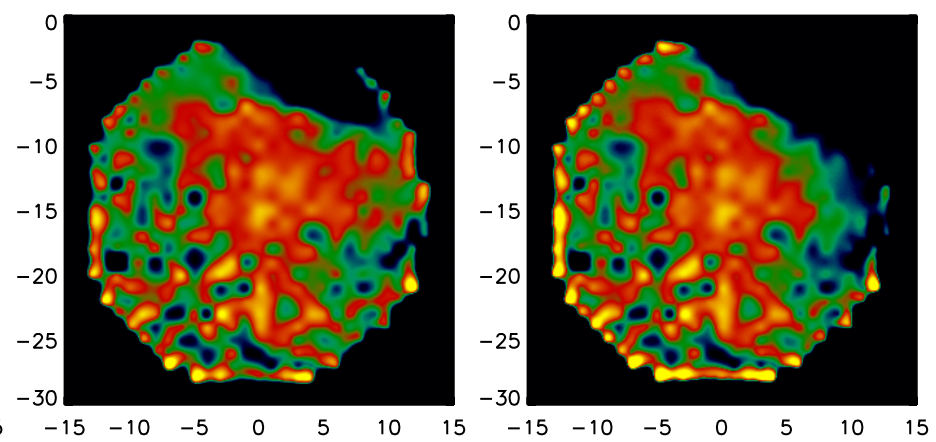

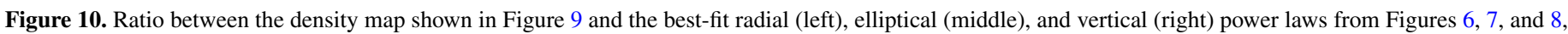
respectively. The color scale label is the logarithm of the ratio. Axes are labeled in kpc from the center of the galaxy.

no systematic trend with azimuthal angle, demonstrating that the halo is consistent with being flattened in the same sense as the disk. The vertical feature at $x=0$, which is an artifact of the chip gap, is very apparent, along with smaller-scale variation. In the right-hand panel, the vertical power-law fit is used; given the similarity between the elliptical and vertical geometry, it is not surprising that this panel shows similar features to the middle panel. However, there is a small systematic negative trend on the right side of the image, which again suggests that a power-law halo is a better description than an exponential thick disk.

\subsection{GHOSTS Fields}

The overlap between the GHOSTS fields and the IMACS data provides a check on our methodology. The density of TRGB stars brighter than $I<24.25$ in the $H S T /$ ACS GHOSTS data is $\log N_{\mathrm{TRGB}}=5.96$ and $\log N_{\mathrm{TRGB}}=6.13$ for Fields 9 and 10 , respectively, while the derived densities from the IMACS data over the identical areas of sky are $\log N_{\mathrm{TRGB}}=5.73$ and $\log N_{\text {TRGB }}=5.92$, factors of 1.7 and 1.6 lower, respectively. While these factors are not unity, they are reassuringly close given the dramatic difference in image quality and depth between the HST/ACS and IMACS data, and validates the use of moderate ground-based data to study stellar halos of galaxies beyond the Local Group.

The factor of $\sim 1.7(\sim 0.2$ dex $)$ provides an indication of the magnitude of uncertainty that is introduced by using groundbased data. Because the factor is similar in both fields, it is not a random error but must reflect a systematic uncertainty, and dominates over the random Poisson error (which is denoted by the error bars of Figures 6-8). With only two overlapping $H S T$ fields, which are both located at similar densities, we cannot determine whether this systematic reflects an overall normalization error, or is specific to the highest density regions. Overlapping HST data at lower stellar densities will be required to disentangle these possible effects. Although there is a small magenta region in Figure 9 within the GHOSTS Field 10 pointing, indicating that the IMACS data give only a lower limit at that location, the lack of magenta within the Field 9 pointing indicates that this is not the cause of the offset.

Note that although the photometric accuracy of the IMACS data is insufficient to determine metallicity via the color of the RGB, Radburn-Smith et al. (2011) report an [Fe/H] estimate based on the TRGB colors of $-1.1 \pm 0.3$ for the HST/ACS GHOSTS data in both of these fields.

$$
\text { 4.4. Shelf }
$$

As noted in Section 4.2, an overdensity of stars is seen on the south side of the galaxy. A rough outline of the region is shown

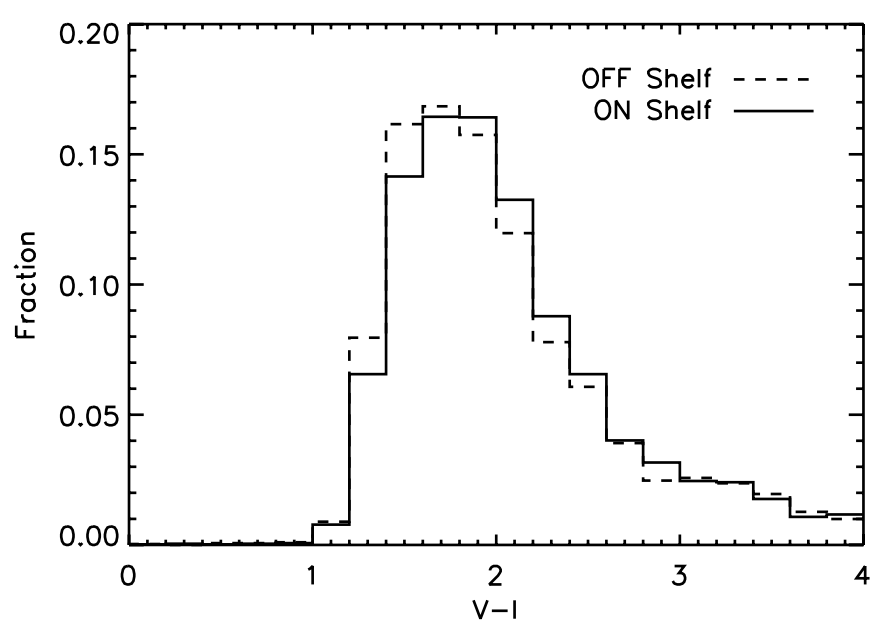

Figure 11. Histograms of the $V-I$ color distribution of stars at the TRGB in GHOSTS Fields 9 ("OFF Shelf") and 10 ("ON Shelf").

with the dashed curve in Figure 9. This overdensity coincides with the optical shelf seen in deep images of Malin \& Hadley (1997). Our detection of this feature demonstrates that it is due to stars, rather than synchrotron emission (as suggested by Beck et al. 1982).

Knowledge of the stellar content in the shelf would greatly aid in understanding its origin. Unfortunately, the high density in this region results in significant blending in the IMACS data, and therefore errors in the stellar colors that are too large to allow the detection of stellar population differences. However, the locations of the GHOSTS pointings are fortuitous: Field 10 lies on the shelf, while Field 9 lies just off the shelf. These fields are at almost identical elliptical radii, so we would expect the stellar populations in these fields to be very similar if the halo were smooth. We can therefore compare the colors of stars detected in the HST/ACS data in these fields to constrain the stellar population of the shelf.

We have plotted the fraction of TRGB stars $(I<24.25)$ as a function of their $V-I$ color for the two GHOSTS fields in Figure 11. The distribution is very similar, indicating that the stellar populations in the shelf are not dramatically different from the rest of the halo at this distance. The differences that are seen are in the sense that the shelf contains more red $(1.8<V-I<2.6)$ TRGB stars, and fewer blue $(1.2<V-I<1.8)$ TRGB stars. Using the transformation between metallicity and TRGB color in Bellazzini et al. (2001), this corresponds to more stars at $-1.3<[\mathrm{Fe} / \mathrm{H}]<-0.7$ and fewer at $[\mathrm{Fe} / \mathrm{H}]<-1.3$. Indeed, using the same data, 
Radburn-Smith et al. (2011) find that the Field 10 TRGB is 0.06 mag redder and 0.05 dex more metal-rich than Field 9.

A higher metallicity in the shelf is consistent with hierarchical models of stellar halo formation, which predict that the highest surface brightness substructures, having been formed from the largest satellites, are more metal-rich than the well-mixed smooth component (Font et al. 2008).

\section{HALO LUMINOSITY AND MASS}

\subsection{Synthetic CMD Calibration}

In order to translate the number of TRGB stars to a total luminosity of the underlying stellar population, we used the online IAC-STAR ${ }^{9}$ synthetic CMD code (Aparicio \& Gallart 2004) to generate an old metal-poor stellar population, and then compared the number of TRGB stars to the total luminosity. We tested several sets of parameters for the stellar population: a single burst lasting for $1 \mathrm{Gyr}$ that ended 12 Gyr or $9 \mathrm{Gyr}$ ago, and a metallicity of $Z=0.0007 \approx 1 / 30 Z_{\odot}, Z=0.002 \approx$ $1 / 10 Z_{\odot}$, or $Z=0.007 \approx 1 / 3 Z_{\odot}$, in all cases using a Kroupa et al. (1993) initial mass function, the Girardi et al. (2000) stellar evolution library, and bolometric corrections based on the Castelli \& Kurucz (2004) models. The synthetic CMDs contained between 10,000 and 20,000 stars above $M_{I}<-3.46$ (equivalent to our TRGB cut at an observed magnitude of $I<24.25)$. The resulting stellar populations had a total integrated luminosity per TRGB star of between $1.2 \times 10^{4}$ and $1.5 \times 10^{4} L_{\odot} / N_{\mathrm{TRGB}}$, increasingly monotonically with metallicity but having very little dependence on the age of the population ( $L / N_{\text {TRGB }}$ is $\approx 3 \%$ higher for the older population). We adopt a scaling of $1.3 \times 10^{4} L_{\odot} / N_{\mathrm{TRGB}}$, appropriate for an old population with metallicity $Z=0.1 Z_{\odot}$, close to the inferred metallicity in the overlapping GHOSTS fields (Radburn-Smith et al. 2011), and caution that large differences between the assumed and intrinsic metallicities could introduce $\sim 20 \%$ systematic errors in the derived luminosities.

Translation to total stellar mass is more problematic due to a numerical issue in the IAC-STAR code that, for some sets of input parameters, gives an $\mathrm{NaN}$ result for some integrated quantities of the stellar population, such as extant stellar mass (A. Aparicio 2010, private communication). Using the stellar mass when available, and extrapolating those mass-to-light ratio trends with metallicity and age to those sets of parameters for which the stellar mass was unavailable, we find that the total stellar mass per TRGB star is between $1.2 \times 10^{4}$ and $2.1 \times$ $10^{4} M_{\odot} / N_{\text {TRGB }}$, increasing as a function of both metallicity and age. We adopt a scaling of $1.6 \times 10^{4} M_{\odot} / N_{\text {TRGB }}$, and again caution that different stellar populations could introduce systematic errors in the derived masses of order $\sim 50 \%$.

\subsection{Halo Luminosity and Mass}

The total number of TRGB stars we derive in our field is $N_{\text {TRGB }}=3.5 \times 10^{4}$, resulting in a minimum halo luminosity of $L_{\text {halo }}>4.6 \times 10^{8} L_{\odot}$ and minimum halo mass of $M_{\text {halo }}>$ $5.6 \times 10^{8} M_{\odot}$. We can estimate the total halo luminosity several ways. First, we note that we have covered approximately $30 \%$ of the area of the galaxy to a distance of $30 \mathrm{kpc}$, and that the halo density drops sufficiently steeply that the majority of the stars should be contained within $30 \mathrm{kpc}$, resulting in a total of $L_{\text {halo }}=1.5 \times 10^{9} L_{\odot}$ and $M_{\text {halo }}=1.9 \times 10^{9} M_{\odot}$. Another estimate of the total luminosity can be determined from the

\footnotetext{
9 http://iac-star.iac.es/iac-star/
}

elliptical power-law fit to the TRGB radial number density; however, as the slope of the projected density is significantly more negative than -1 , the integral diverges at small radius. We have therefore imposed an inner cutoff at a radius along the minor axis of $5 \mathrm{kpc}$, and caution that this estimate does not account for most of the halo stars that overlap with the galactic disk. The total halo luminosity using these assumptions is $L_{\text {halo }}=(2.6 \pm 0.3) \times 10^{9} L_{\odot}$, while the halo mass is $M_{\text {halo }}=(3.2 \pm 0.4) \times 10^{9} M_{\odot}$. The main difference between these two estimates is due to small-scale structure, rather than the counts beyond $30 \mathrm{kpc}$ which only account for $15 \%$ of the luminosity using the power-law fit. The quoted random errors, which are due to the uncertainty in the power-law fit, certainly underestimate the true uncertainty; for example, systematically shifting the inner points by the estimated systematic uncertainty of 0.2 dex, as in Section 4.1, changes the luminosity and mass by $50 \%$. The confluence of these various estimates suggests that the total halo luminosity is $L_{\text {halo }} \approx(2 \pm 1) \times 10^{9} L_{\odot}$ and the stellar mass is $M_{\text {halo }} \approx(2.5 \pm 1.5) \times 10^{9} M_{\odot}$.

We estimate the fraction of the total light and stellar mass of the galaxy contained in the halo from the total RC3 (de Vaucouleurs et al. 1991) $B$ and $V$ magnitudes of $B=8.04$, and $V=7.19$, which gives an absolute magnitude of $M_{V}=-20.6$ and a $V$-band luminosity of $\approx 1.5 \times 10^{10} L_{\odot}$. This corresponds to a total stellar mass of $\approx 4.4 \times 10^{10} M_{\odot}$, assuming $M / L_{V}=2.9$, as given by the $B-V$ versus $M / L_{V}$ relation in Bell \& de Jong (2001). Therefore, a fraction $\sim 0.13$ of the luminosity and $\sim 0.06$ of the stellar mass of NGC 253 is in the form of its halo. This is consistent with theoretical models that predict that the median galaxy at this luminosity contains a stellar halo with $1 \%-5 \%$ of the galaxy stellar mass (Purcell et al. 2007).

The only other galaxies for which the total luminosity and mass of the stellar halo can be obtained are the Milky Way and M31, both of which are of similar luminosity to NGC 253. Measurements place the total luminosity and mass of the Milky Way's stellar halo at $L_{V} \sim 10^{9} L_{\odot}$ and $M \sim 2 \times 10^{9} M_{\odot}$, respectively (see the summary in Bullock \& Johnston 2005), and the luminosity of the M31 stellar halo at $L \sim 10^{9} L_{\odot}$ (Ibata et al. 2007), similar in magnitude to but somewhat smaller than that of NGC 253.

\section{CONCLUSIONS}

We have detected resolved RGB stars in the stellar halo of NGC 253 in both HST/ACS and ground-based Magellan/ IMACS imaging data. The clean HST data are only able to probe a small area of the halo, but provide a benchmark by which we can evaluate the ground-based data. The large area covered by a single IMACS pointing provides a global picture of the halo, reaching out to distances of $30 \mathrm{kpc}$ from the center of the galaxy, which is required given the substructure and largescale features predicted in models of stellar halo formation and seen in the observations.

While previous studies of resolved stellar halos have successfully detected stars in the halo and discovered spectacular tidal features (Mouhcine et al. 2010), only for the Milky Way and M31 has there been significant quantitative analysis of the halo profile and structure. In this work, we have performed detailed artificial star tests in order to make quantitative measurements that can be compared to theoretical predictions and compared to observations of other galaxies.

The projected stellar density declines with elliptical radius as a power law of slope $-2.8 \pm 0.6$. This is consistent with, but at the steep end, of the density profiles found for the Milky Way 
Table 1

Positions and Magnitudes of Detected Stars

\begin{tabular}{lcccc}
\hline \hline ID & RA & Dec & $V$ & $I$ \\
\hline 1 & 004829.81 & -253159.5 & 25.79 & 24.95 \\
2 & 004829.78 & -253047.4 & 26.57 & 24.36 \\
3 & 004829.77 & -253141.2 & 25.32 & 23.85 \\
4 & 004829.76 & -253146.3 & 28.29 & 24.48 \\
5 & 004829.73 & -253123.7 & 26.76 & 24.32 \\
\hline
\end{tabular}

(This table is available in its entirety in machine-readable form in the online journal. A portion is shown here for guidance regarding its form and content.)

and M31 halos; for example, Bell et al. (2008) find that the threedimensional density falls off as a power of index of between -2 and -4 , corresponding to projected radial power-law indices of -1 to -3 , and the projected radial profile of the halo of M31 has a power-law index of $\approx-2$ (Ibata et al. 2007). An exponential vertical distribution is also found to provide an acceptable fit, with an extraordinarily large-scale height of $h_{z}=5.3 \pm$ $0.8 \mathrm{kpc}$. However, interpretation of the observed structure as a thick disk rather than a power-law halo would require an unrealistically large vertical velocity dispersion, and is inconsistent with the observed halo density in the HST/GHOSTS fields.

The halo is found to be flattened in the same direction as the stellar disk, with a projected axis ratio of $\approx 0.35 \pm 0.1$. This is consistent with what has been found for other galaxies: the Milky Way halo is also flattened in the same sense as its disk at small radii (e.g., Carollo et al. 2007), de Jong et al. (2009) find a typical halo axis ratio of $c / a \approx 0.4$ based on $H S T /$ GHOSTS data of nearly edge-on galaxies, and although not quantitative, the star counts in the halo of NGC 891 also appear significantly flattened like the disk (Mouhcine et al. 2010). The dark matter halo that dominates the potential is also predicted in galaxy formation simulations to be flattened in the same sense as the galactic disk within $10 \%$ of the virial radius (Bailin et al. 2005). Observations of more galaxies are required to see if this is a universal feature of stellar halos.

Davidge (2010), who detected AGB stars in the halo of NGC 253, found their distribution to be flattened similarly to what is found in this study for the older RGB stars. Unfortunately, our observations are not sensitive to these younger stars, and so further observations will be required in order to fully understand the relationship between these populations.

Density maps of the halo structure show substructure on a variety of scales. The shelf-like feature seen in earlier deep photographic studies (Malin \& Hadley 1997) is evident. In addition, there is a significant amount of smaller kpc-scale structure. These features are not apparent in the small HST fields but require the full IMACS field of view to appreciate.

We estimate the total luminosity of the stellar halo to be $\sim(2 \pm$ 1) $\times 10^{9} L_{\odot}$, and the stellar mass to be $\sim(2.5 \pm 1.5) \times 10^{9} M_{\odot}$. This corresponds to a fraction 0.13 of the total luminosity and 0.06 of the total stellar mass of the galaxy. These values are consistent with the theoretical models of Purcell et al. (2007). The NGC 253 halo luminosity is a factor of $\approx 2$ larger than the halos of the Milky Way and M31, despite the similar total luminosity of these galaxies, indicating significant galaxy-togalaxy scatter in the relative halo fraction, as predicted by models.

This paper provides a roadmap for analyzing ground-based imaging data of the stellar halos of galaxies beyond the Local Group in a quantitative way. These techniques will be applied in future work to a series of other galaxies for which we have obtained similar data. The resulting collection of quantitative global measurements of the scale and structure of stellar halos of a broad sample of galaxies will allow for statistical comparison to models of stellar halo formation, and will provide vital constraints on the galaxy assembly processes that are traced by the halos.

A catalog of the detected sources is shown in Table 1 . The same data is also available at http://www.astro.lsa.umich.edu/ $\sim$ jbailin/ngc253/ and on the Centre de Données astronomiques de Strasbourg (CDS).

We thank Anna Gallazzi for helping obtain the Magellan observations and Chien Peng for useful data reduction advice. This work has made use of the IAC-STAR Synthetic CMD computation code. IAC-STAR is supported and maintained by the computer division of the IAC. This research has made use of the NASA/IPAC Extragalactic Database (NED) which is operated by the Jet Propulsion Laboratory, California Institute of Technology, under contract with the National Aeronautics and Space Administration. This work was supported by the National Science Foundation through grant AST-1008342, and by HST grant GO-11613. Support for Program number GO-11613 was provided by NASA through a grant from the Space Telescope Science Institute, which is operated by the Association of Universities for Research in Astronomy, Inc., under NASA contract NAS5-26555.

Facilities: Magellan:Baade (IMACS), HST (ACS)

\section{REFERENCES}

Aparicio, A., \& Gallart, C. 2004, AJ, 128, 1465

Bailin, J., et al. 2005, ApJ, 627, L17

Beck, R., Hutschenreiter, G., \& Wielebinski, R. 1982, A\&A, 106, 112

Bell, E. F., \& de Jong, R. S. 2001, ApJ, 550, 212

Bell, E. F., Xue, X. X., Rix, H., Ruhland, C., \& Hogg, D. W. 2010, AJ, 140, 1850

Bell, E. F., et al. 2008, ApJ, 680, 295

Bellazzini, M., Ferraro, F. R., \& Pancino, E. 2001, ApJ, 556, 635

Belokurov, V., et al. 2007, ApJ, 657, L89

Binney, J., \& Tremaine, S. 2008, Galactic Dynamics (2nd ed.; Princeton, NJ: Princeton Univ. Press)

Bullock, J. S., \& Johnston, K. V. 2005, ApJ, 635, 931

Bullock, J. S., Kravtsov, A. V., \& Weinberg, D. H. 2001, ApJ, 548, 33

Carollo, D., et al. 2007, Nature, 450, 1020

Castelli, F., \& Kurucz, R. L. 2004, in IAU Symp. 210, Modelling of Stellar Atmospheres, ed. N. Piskunov, W. W. Weiss, \& D. F. Gray (Cambridge: Cambridge Univ. Press), A20

Chapman, S. C., Ibata, R., Lewis, G. F., Ferguson, A. M. N., Irwin, M., McConnachie, A., \& Tanvir, N. 2006, ApJ, 653, 255

Collins, M. L. L., et al. 2011, MNRAS, 413, 1548

Comerón, F., Torra, J., Méndez, R. A., \& Gómez, A. E. 2001, A\&A, 366, 796

Cooper, A. P., Cole, S., Frenk, C. S., \& Helmi, A. 2010a, MNRAS, submitted (arXiv:1011.1926)

Cooper, A. P., et al. 2010b, MNRAS, 406, 744

Davidge, T. J. 2010, ApJ, 725, 1342

de Jong, R. S. 2008, MNRAS, 388, 1521

de Jong, R. S., Radburn-Smith, D. J., \& Sick, J. N. 2009, arXiv:0904.2777

de Jong, R. S., et al. 2007, ApJ, 667, L49

de Vaucouleurs, G., de Vaucouleurs, A., Corwin, H. G., Buta, R. J., Paturel, G., \& Fouque, P. 1991, Third Reference Catalogue of Bright Galaxies (Vol. 1-3; Berlin: Springer)

Durrell, P. R., Sarajedini, A., \& Chandar, R. 2010, ApJ, 718, 1118

Fitzgibbons, G. L. 1990, PhD thesis, Univ. Florida

Font, A. S., Johnston, K. V., Ferguson, A. M. N., Bullock, J. S., Robertson,

B. E., Tumlinson, J., \& Guhathakurta, P. 2008, ApJ, 673, 215

Forbes, D. A., \& Depoy, D. L. 1992, A\&A, 259, 97

Franke, R. 1982, Comput. Math. Appl., 8, 273

Gawiser, E., et al. 2006, ApJS, 162, 1

Girardi, L., Bressan, A., Bertelli, G., \& Chiosi, C. 2000, A\&AS, 141, 371 
Helmi, A., White, S. D. M., de Zeeuw, P. T., \& Zhao, H. 1999, Nature, 402, 53 Ibata, R., Chapman, S., Ferguson, A. M. N., Lewis, G., Irwin, M., \& Tanvir, N. 2005, ApJ, 634, 287

Ibata, R., Martin, N. F., Irwin, M., Chapman, S., Ferguson, A. M. N., Lewis, G. F., \& McConnachie, A. W. 2007, ApJ, 671, 1591

Jerjen, H., Freeman, K. C., \& Binggeli, B. 1998, AJ, 116, 2873

Johnston, K. V., Bullock, J. S., Sharma, S., Font, A., Robertson, B. E., \& Leitner, S. N. 2008, ApJ, 689, 936

Kalirai, J. S., et al. 2006, ApJ, 648, 389

Karachentsev, I. D., et al. 2003, A\&A, 404, 93

Koribalski, B. S., et al. 2004, AJ, 128, 16

Kroupa, P., Tout, C. A., \& Gilmore, G. 1993, MNRAS, 262, 545

Majewski, S. R., Skrutskie, M. F., Weinberg, M. D., \& Ostheimer, J. C. 2003, ApJ, 599, 1082

Malin, D., \& Hadley, B. 1997, PASA, 14, 52

Martínez-Delgado, D., Peñarrubia, J., Gabany, R. J., Trujillo, I., Majewski, S. R., \& Pohlen, M. 2008, ApJ, 689, 184

Martínez-Delgado, D., et al. 2010, AJ, 140, 962
McConnachie, A. W., et al. 2009, Nature, 461, 66

Monet, D., et al. 1998, VizieR Online Data Catalog, I/252

Mouhcine, M., Ibata, R., \& Rejkuba, M. 2010, ApJ, 714, L12

Pence, W. D. 1980, ApJ, 239, 54

Purcell, C. W., Bullock, J. S., \& Zentner, A. R. 2007, ApJ, 666, 20

Radburn-Smith, D. J., et al. 2011, ApJ, submitted

Radovich, M., Kahanpää, J., \& Lemke, D. 2001, A\&A, 377, 73

Rejkuba, M., Greggio, L., Harris, W. E., Harris, G. L. H., \& Peng, E. W. 2005, ApJ, 631, 262

Searle, L., \& Zinn, R. 1978, ApJ, 225, 357

Sirianni, M., et al. 2005, PASP, 117, 1049

Tanaka, M., Chiba, M., Komiyama, Y., Guhathakurta, P., Kalirai, J. S., \& Iye, M. 2010, ApJ, 708, 1168

White, S. D. M., \& Rees, M. J. 1978, MNRAS, 183, 341

Xue, X., et al. 2010, ApJ, submitted, arXiv:1011.1925

Yanny, B., et al. 2003, ApJ, 588, 824

Yoachim, P., \& Dalcanton, J. J. 2006, AJ, 131, 226

Zheng, Z., et al. 1999, AJ, 117, 2757 